\title{
Efficient Photocatalytic Degradation of Rhodamine B Dye by Aligned Arrays of Self-Assembled Hydrogen Titanate Nanotubes
}

\author{
Sriparna Chatterjee, ${ }^{1,2}$ Avesh K. Tyagi, ${ }^{3}$ and Pushan Ayyub ${ }^{1}$ \\ ${ }^{1}$ Department of Condensed Matter Physics \& Materials Science, Tata Institute of Fundamental Research, Mumbai 400005, India \\ ${ }^{2}$ Colloids and Materials Chemistry Department, Institute of Minerals and Materials Technology (CSIR), Bhubaneswar 751013, India \\ ${ }^{3}$ Chemistry Division, Bhabha Atomic Research Centre, Mumbai 400085, India
}

Correspondence should be addressed to Pushan Ayyub; pushan@tifr.res.in

Received 27 November 2013; Revised 25 March 2014; Accepted 25 March 2014; Published 15 April 2014

Academic Editor: Takuya Tsuzuki

Copyright (C) 2014 Sriparna Chatterjee et al. This is an open access article distributed under the Creative Commons Attribution License, which permits unrestricted use, distribution, and reproduction in any medium, provided the original work is properly cited.

\begin{abstract}
We show that an aligned array of hydrothermally grown, multiwalled hydrogen titanate $\left(\mathrm{H}_{2} \mathrm{Ti}_{3} \mathrm{O}_{7}\right)$ nanotubes-anchored to both faces of a metallic Ti foil-acts as an efficient photocatalyst. We studied the degradation of rhodamine $\mathrm{B}$ dye in the presence of the nanostructured photocatalyst under UV irradiation, by monitoring the optical absorption of the dye. Rhodamine B was chosen as a representative - and particularly harmful-industrial pollutant dye. The inner and outer diameters of the $\mathrm{H}_{2} \mathrm{Ti}_{3} \mathrm{O}_{7}$ nanotubes were $5 \mathrm{~nm}$ and $10 \mathrm{~nm}$, respectively. The nanotube array catalyst is recyclable and structurally stable. Most importantly, it shows comparable or higher photodecomposition rate constant than those of both $\mathrm{H}_{2} \mathrm{Ti}_{3} \mathrm{O}_{7}$ nanotube powder and P-25 (Degussa). The enhanced photocatalytic performance may be ascribed to the nanotube array having a superhydrophilic surface with a high accessible surface area.
\end{abstract}

\section{Introduction}

A prime issue in environmental remediation [1] is to remove dyes and other commercial colorants from wastewater effluents of textile mills and other dye processing units, because $15 \%$ of synthetic textile dyes are believed to get dispersed in wastewater during manufacturing and processing [2]. Among the best agents for dye degradation are wide band gap semiconductors [3], in which electron-hole pairs are formed upon photoexcitation (usually in the UV). A fraction of these get trapped in shallow defect states and are subsequently available for relatively slow, redox processes [4]. Most organic photodegradation reactions utilize the oxidizing power of holes and result in the conversion of the organic species at the interfaces into nontoxic end products. An ideal photocatalyst should be stable, inexpensive, nontoxic, and highly photoactive, and the redox potential of the $\mathrm{H}_{2} \mathrm{O} /{ }^{\circ} \mathrm{OH}$ couple must lie within its band gap. In principle, semiconductors such as $\mathrm{TiO}_{2}, \mathrm{ZnO}, \mathrm{Fe}_{2} \mathrm{O}_{3}, \mathrm{CdS}$, and $\mathrm{ZnS}$ can all act as sensitizers for such light-induced redox processes. In practice, $\mathrm{TiO}_{2}$ (anatase) appears to be the most promising photocatalyst for the destruction of organic pollutants due to its low cost, biological and chemical stability, nonphotocorrosivity, and superior photocatalytic activity $[5,6]$. Further, there are several obvious advantages of having a semiconductor photocatalyst in the nanocrystalline form, such as (a) tunability of the band gap, (b) higher specific surface area, and (c) greater availability of trap states.

Photocatalytic activity has been mostly studied using spherical or equiaxed nanoparticles, in which the catalytic efficiency indeed increases with decreasing particle size [7]. However, the recovery of nanoparticulate catalysts from the solution at the end of the reaction cycle may prove to be impractical. Also, spherical nanoparticles often tend to agglomerate into larger particles, thereby reducing their catalytic activity. Considerable effort has therefore gone into the search for low cost, high activity, and easily recyclable photocatalysts based on titanium oxide [8-10]. An elegant and practical solution to the above issues would be to obtain the photocatalyst in the form of an aligned array of $1 \mathrm{D}$ structures anchored to a substrate. Such a catalyst would be easy to handle and not allow aggregation to occur, 
thus permanently providing a high interfacial area for the reactants.

Among the various 1D nanostructures, nanotubes are now ubiquitous in the world of nanoscience, particularly after the discovery of noncarbon nanotubes. In particular, titanate nanotubes have attracted attention as cathodes for rechargeable Li-ion batteries [11]. However, studies of their photocatalytic properties are not only scarce but also somewhat inconclusive. Hydrogen titanate $\left(\mathrm{H}_{2} \mathrm{Ti}_{3} \mathrm{O}_{7}\right)$ nanotubes have been tried out as a photocatalyst, but only with limited success. For example, Hodos et al. [12] suggested that the $\mathrm{H}_{2} \mathrm{Ti}_{3} \mathrm{O}_{7}$ nanotubes are normally photocatalytically inactive and require to be photosensitized by $\mathrm{CdS}$ nanoparticles. On the other hand, Amano et al. [13] claimed that though titanate crystallites with a small amount of defects show considerable photocatalytic activity-liberating oxygen from an aqueous solution of silver sulphate as well as oxidatively decomposing acetic acid-it is less than that of anatase for the liberation of $\mathrm{H}_{2}$ from methanol. From a detailed study using methylene blue as the dye, Baiju et al. [14] reported a very high surface adsorption of dye molecule with a very low dye degradation rate because of poor crystallinity-for both as-prepared and calcined titanates, in agreement with earlier findings [15]. Note that all the above studies were made with randomly oriented (mesh-like) $\mathrm{H}_{2} \mathrm{Ti}_{3} \mathrm{O}_{7}$ nanotube samples. $\mathrm{H}_{2} \mathrm{Ti}_{3} \mathrm{O}_{7}$ nanotube meshes also show enhanced activity in the gas phase photocatalytic oxidation of ethylene [16].

Here we report the first study of the photocatalytic activity of an aligned array of multiwalled $\mathrm{H}_{2} \mathrm{Ti}_{3} \mathrm{O}_{7}$ nanotubes anchored to a substrate. This geometry provides comparatively greater exposure of the sample surface to the reacting environment than that of a random collection of nanotubes. We have synthesized a roughly aligned array of $\mathrm{H}_{2} \mathrm{Ti}_{3} \mathrm{O}_{7}$ nanotubes by a hydrothermal technique and studied its use for the photochemical degradation of the rhodamine $\mathrm{B}(\mathrm{RhB})$ dye under UV light exposure. The catalytic efficiency of this material has been benchmarked against a standard industrial photocatalyst (P-25). We point out that RhB is accepted as a representative dye pollutant for such studies. While most dyes are resistant to biodegradation and direct photolysis, nitrogen-containing dyes such as $\mathrm{RhB}$ are particularly dangerous as they undergo a natural anaerobic degradation to yield potentially carcinogenic aromatic amines [17].

\section{Materials and Methods}

2.1. Hydrothermal Synthesis of Multiwalled Tri-Titanate Nanotubes. A modified hydrothermal synthesis route was used to obtain self-assembled, multiwalled $\mathrm{H}_{2} \mathrm{Ti}_{3} \mathrm{O}_{7}$ nanotube arrays (NTA) on oxidized Ti foils [18]. Typically, a $30 \mathrm{~mm} \times 5 \mathrm{~mm}$ $\times 25 \mu \mathrm{m}$ Ti foil (Sigma-Aldrich, 99.9\%) was first oxidized in air at $800^{\circ} \mathrm{C}$ for $5 \mathrm{~h}$, then dipped in $35 \mathrm{~mL}$ of $10 \mathrm{M} \mathrm{NaOH}$ in a sealed Teflon reactor, and autoclaved at $200^{\circ} \mathrm{C}$ for $6 \mathrm{~h}$ under an autogenous pressure. After cooling down to room temperature, the foils were thoroughly washed with $1: 10$ $\mathrm{HCl}$ (by volume)-typically for $\approx 10-12 \mathrm{~h}$-till the $\mathrm{pH}$ of the wash became $\approx 6.5$. Finally the samples were washed with hot deionized water to remove any traces of $\mathrm{NaCl}$ and then dried at $70^{\circ} \mathrm{C}$. The catalytic efficiency of the asprepared $\mathrm{H}_{2} \mathrm{Ti}_{3} \mathrm{O}_{7}$ nanotube array sample was compared with that of an unsupported $\mathrm{H}_{2} \mathrm{Ti}_{3} \mathrm{O}_{7}$ nanotube powder or mesh, which was synthesized from bulk anatase powder (Sigma, 99.999\%) and $10 \mathrm{M} \mathrm{NaOH}$ using a hydrothermal process [19]. The results were benchmarked by comparing with the performance of a standard $\mathrm{TiO}_{2}$-based photocatalyst: Aeroxide P-25 Degussa with an average primary particle size $=21 \mathrm{~nm}$ and specific surface area $(B E T)=50 \pm 15 \mathrm{~m}^{2} \mathrm{~g}^{-1}$.

2.2. Structural Characterization. The crystallographic phase of the as-prepared $\mathrm{H}_{2} \mathrm{Ti}_{3} \mathrm{O}_{7}$ nanotubes was investigated using a Panalytical X'Pert Pro powder X-ray diffractometer (XRD) using $\mathrm{Cu}-\mathrm{K}_{\alpha}$ radiation, while their microstructure was studied using an eLine field emission scanning electron microscope (FESEM) operated at $20 \mathrm{kV}$. Energy dispersive $\mathrm{X}$-ray (EDX) analysis was carried out to obtain the elemental composition. The morphology and crystal structure of individual nanotubes were studied using a FEI Tecnai-20 transmission electron microscope (TEM), equipped with a $\mathrm{LaB}_{6}$ filament, and operated at $200 \mathrm{kV}$. Samples for TEM were prepared by scraping the nanotubes off the foil, dispersing in ethanol, and placing a drop of the dispersion on a $\mathrm{Cu}$ grid covered with a holey carbon film, which was then dried. The surface area and pore size distribution were obtained using the Brunauer-Emmet-Teller (BET) from $\mathrm{N}_{2}$ physisorption data at $77 \mathrm{~K}$ recorded using a Micromeritics ASAP 2020 analyzer. About $100 \mathrm{mg}$ of each sample was degassed at $10^{-6}$ Torr and $100^{\circ} \mathrm{C}$ prior to $\mathrm{N}_{2}$ adsorption. The BarretJoyner-Halenda (BJH) method was employed to obtain pore size distribution. Diffuse reflectance UV-visible spectra were recorded on a JASCO V-670 spectrophotometer, using $\mathrm{BaSO}_{4}$ as a reference.

2.3. Photocatalytic Activity. We examined the photocatalytic performance of the substrate-supported $\mathrm{H}_{2} \mathrm{Ti}_{3} \mathrm{O}_{7}$ NTA, the unsupported $\mathrm{H}_{2} \mathrm{Ti}_{3} \mathrm{O}_{7}$ nanotube powder (for comparison), the oxidized $\mathrm{Ti}$ foil precursor (for comparison), and $\mathrm{P}$ 25 powder (as a standard reference) via the photo-assisted decolorization of an aqueous solution of $\mathrm{RhB}$ dye under UV irradiation $(\lambda=365 \mathrm{~nm})$ at room temperature $(23 \pm$ $2^{\circ} \mathrm{C}$ ). Four $6 \mathrm{~W} \mathrm{UV}$ lamps were used as the light source. Approximately $10 \mathrm{mg}$ of the $\mathrm{H}_{2} \mathrm{Ti}_{3} \mathrm{O}_{7}$ NTA sample (inclusive of the substrate) was suspended horizontally in $10 \mathrm{~mL}$ of 1 $\times 10^{-6} \mathrm{M}$ RhB solution in a $25 \mathrm{~mL}$ Pyrex beaker equipped with a magnetic stirrer. The beaker was tightly covered during the experiment to prevent evaporative losses. The net volumetric loss during the full experiment was in the range of $2-3 \%$. Before UV irradiation, the dye solution was stirred in the dark for $6 \mathrm{~h}$ to ensure adsorption-desorption equilibrium of the dye on the catalyst surface so that the loss of the dye due to adsorption is correctly accounted for. The zero time reading was obtained from a blank solution kept in the dark but otherwise treated similarly to the irradiated sample. During illumination, about $1 \mathrm{~mL}$ of the dye solution was extracted after each 30-45 minute interval, and optical absorption spectra were recorded to determine the degradation rate (decrease in absorption intensity versus 
irradiation time) of $\mathrm{RhB}$. The test solution was returned to the beaker each time after the absorption measurement to maintain a constant catalyst: reactant ratio throughout the experiment. The characteristic optical absorption peak of $\mathrm{RhB}$ at $553 \mathrm{~nm}$ was chosen to monitor the photo-induced degradation. The procedure was repeated for a second cycle to examine the stability and applicability of $\mathrm{H}_{2} \mathrm{Ti}_{3} \mathrm{O}_{7}$ nanotube array samples for multiple uses. Blank experiments without addition of catalyst and with the catalyst in the dark were also carried out.

\section{Results and Discussion}

3.1. Structure and Morphology. We have earlier reported a detailed, electron microscopic study of each stage of the growth of the $\mathrm{H}_{2} \mathrm{Ti}_{3} \mathrm{O}_{7}$ nanotube array [18], the significant steps of which are as follows. It is important to note that the oxidized Ti foil (precursor) actually consists of a central, virtually unoxidized $\mathrm{Ti}$ layer sandwiched between layers of $\mathrm{TiO}_{2}$ (rutile). During hydrothermal treatment, nascent nanotubes were first detected at the $\mathrm{Ti} / \mathrm{TiO}_{2}$ interface. This indicates that the Ti-rich core layer acts as a substrate for the growth of the tri-titanate nanotube array, whereas the surrounding oxide (rutile) layers serve as the source material. Complete dissolution of the rutile phase takes about six hours, and the resulting $1 \mathrm{D}_{2} \mathrm{Ti}_{3} \mathrm{O}_{7}$ nanostructures grow to a length of about $45-50 \mu \mathrm{m}$ on either side of the central Ti foil. High resolution TEM studies of the nature of the nanotube walls indicate that the tube formation takes place by a "scrolling" of the multilayer nanosheets. Hydrothermal treatment for $6 \mathrm{~h}$ completely converts the rutile layers to a monoclinic, tri-titanate $\left(\mathrm{H}_{2} \mathrm{Ti}_{3} \mathrm{O}_{7}\right)$ phase, as shown by their respective X-ray diffractograms (Figures 1(a) and 1(b), resp.). Figure 1 (c) shows the XRD pattern of free $\mathrm{H}_{2} \mathrm{Ti}_{3} \mathrm{O}_{7}$ nanotube powder dispersed on a glass substrate. Unlike the substrate-attached $\mathrm{H}_{2} \mathrm{Ti}_{3} \mathrm{O}_{7}$, which is partially oriented along [200], the nanotube powder is unoriented. EDX spectra (not shown here) indicate the presence of only $\mathrm{Ti}$ and $\mathrm{O}$, and no sodium was detected. Cross-sectional SEM images recorded at a tilt angle of $89^{\circ}$ (Figure 2(a)) clearly show that the $\mathrm{H}_{2} \mathrm{Ti}_{3} \mathrm{O}_{7}$ phase actually consists of a brush-like array of $1 \mathrm{D}$ structures (with an average length $\approx 45 \mu \mathrm{m}$ on both sides) growing outwards from the central, metallic Ti layer. A plan view SEM (Figure 2(b)) shows that these unagglomerated, roughly parallel $1 \mathrm{D}$ structures provide an extremely high effective surface area. A typical TEM image (Figure 2(c)), obtained by dispersing a sample of the scraped material on a $\mathrm{Cu}$ grid, confirms that each $1 \mathrm{D}$ structure is a hollow, multiwalled nanotube with approximate inner and outer diameters of $5 \mathrm{~nm}$ and $10 \mathrm{~nm}$, respectively. The XRD pattern of the $\mathrm{H}_{2} \mathrm{Ti}_{3} \mathrm{O}_{7}$ phase indicates a preferential alignment of the nanotubes along the (200) axis.

Figure 3 shows the typical nitrogen adsorptiondesorption isotherm obtained from an aligned array of $\mathrm{H}_{2} \mathrm{Ti}_{3} \mathrm{O}_{7}$ nanotubes. The nature of the hysteresis in the isotherm at a high relative pressure $\left(p / p_{0}\right)$ range (Type IVIUPAC) indicates the mesoporous character of the sample [20]. The inflection observed at $p / p_{0} \sim 0.9$ may be attributed to the porous structure of the sample, consistent with the SEM micrographs. The BET surface area of the substrate-attached sample used for the experiments is not very high $\left(22.7 \mathrm{~m}^{2} / \mathrm{g}\right)$ due to the mass contributed by the substrate (central Ti-rich layer) to which the nanowires are anchored. The BET surface area for the rutile-coated precursor foil is only $0.1430 \mathrm{~m}^{2} \mathrm{~g}^{-1}$, and no hysteresis loop is observed for this sample. Thus, the conversion from the rutile layer to the $\mathrm{H}_{2} \mathrm{Ti}_{3} \mathrm{O}_{7}$ NTA is accompanied by a 150 -fold increase in the specific surface area. Expectedly, the specific surface area of free $\mathrm{H}_{2} \mathrm{Ti}_{3} \mathrm{O}_{7}$ nanotubes (with the same dimensions as the substrate-attached ones) was found to be in much higher $\left(230-250 \mathrm{~m}^{2} \mathrm{~g}^{-1}\right)[16]$.

3.2. Diffused Reflectance UV-Visible Spectroscopy. The diffuse reflectance spectrum of the $\mathrm{H}_{2} \mathrm{Ti}_{3} \mathrm{O}_{7}$ NTA shows a distinct blue shift with respect to the oxidized $\mathrm{Ti}$ foil precursor (Figure 4). The band gap was calculated for both systems from the optical spectra using the equation, $E_{\text {band-gap }}=$ $h c / \lambda_{\text {int }}$, where " $h$ " is Plank's constant, " $c$ " is the velocity of light, and " $\lambda_{\text {int }}$ " is the wavelength corresponding to the intersection of the extension of linear part of the spectrum and the $x$-axis. The calculated band gap for the $\mathrm{H}_{2} \mathrm{Ti}_{3} \mathrm{O}_{7}$ nanotube sample is $3.4 \mathrm{eV}$, while that for the oxidized $\mathrm{Ti}$ precursor is $2.9 \mathrm{eV}$.

3.3. Photocatalytic Activity. The photocatalytic activities of the aligned $\mathrm{H}_{2} \mathrm{Ti}_{3} \mathrm{O}_{7}$ NTA, the unsupported $\mathrm{H}_{2} \mathrm{Ti}_{3} \mathrm{O}_{7}$ nanotube powder, the oxidized Ti foil precursor, and $\mathrm{P}-25$ powder (as a standard reference) were estimated by studying the photo-induced degradation of the $553 \mathrm{~nm}$ absorption peak of rhodamine $\mathrm{B}$ as the colored contaminant. A time evolution study of the absorption spectrum of RhB solution exposed to UV light in the presence of the $\mathrm{H}_{2} \mathrm{Ti}_{3} \mathrm{O}_{7}$ NTA (Figure 5(a)) clearly shows a steady extinction of the absorption maximum of $\mathrm{RhB}$ with increasing UV exposure time, till it virtually disappears after $10 \mathrm{~h}$ of exposure. After a similar period in the presence of the precursor $\mathrm{Ti}$ oxide foil (with the same dimensions as that of the NTA anchored to the Ti foil), there was only a marginal change in the $\mathrm{RhB}$ absorption profile (Figure 5(b)).

The catalytic performance of the $\mathrm{H}_{2} \mathrm{Ti}_{3} \mathrm{O}_{7}$ NTA was quantitatively benchmarked by carrying out the same reaction with (a) unsupported $\mathrm{H}_{2} \mathrm{Ti}_{3} \mathrm{O}_{7}$ nanotube powder and (b) a standard photocatalyst such as P-25, which is a mixture of nanocrystalline rutile and anatase. For this, it is essential to have a fairly accurate knowledge of the mass of the $\mathrm{H}_{2} \mathrm{Ti}_{3} \mathrm{O}_{7}$ nanotubes anchored to the substrate. We found that the mass of nanorods physically scraped off from the surface was subject to some degree of inaccuracy because either the nanotubes were not completely removed, or a part of the substrate also tended to get scraped off. A relatively more accurate method is to estimate the number of nanotubes per unit area from a statistically significant number of SEM images and calculate the total mass per unit area of substrate by assuming theoretical density of $\mathrm{H}_{2} \mathrm{Ti}_{3} \mathrm{O}_{7}\left(3.163 \mathrm{~g} / \mathrm{cm}^{3}\right)$. By scaling appropriately to the size of the experimental nanotube 


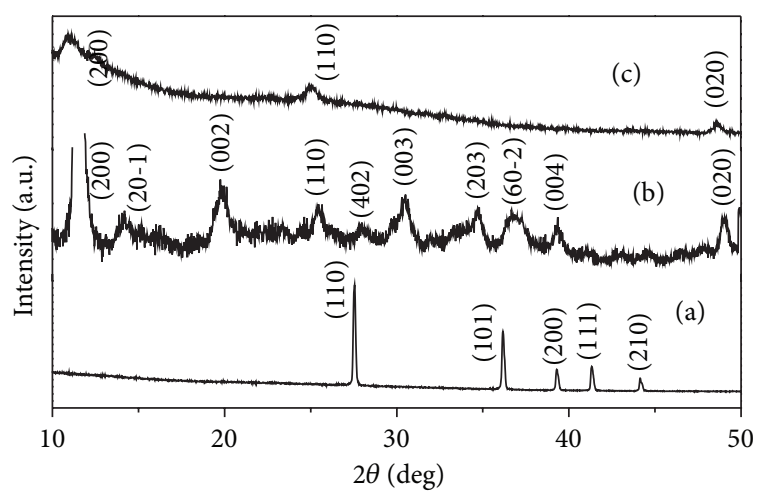

FIGURE 1: X-ray diffraction patterns of the (a) precursor oxidized Ti foil showing the pure rutile phase of $\mathrm{TiO}_{2}$, (b) aligned $\mathrm{H}_{2} \mathrm{Ti}_{3} \mathrm{O}_{7}$ nanotube array showing preferential growth along $<200>$ direction of the monoclinic tri-titanate phase, and (c) free $\mathrm{H}_{2} \mathrm{Ti}_{3} \mathrm{O}_{7}$ nanotube powder.

array sample $(30 \mathrm{~mm} \times 5 \mathrm{~mm})$ and assuming nanotubes of uniform inner and outer diameter growing outwards from both faces of the Ti substrate, we obtained the total mass of the $\mathrm{H}_{2} \mathrm{Ti}_{3} \mathrm{O}_{7}$ nanotubes per sample with an estimated error of $\pm 10 \%$.

In each case, the time evolution of the optical absorption was used to calculate the photodecomposition rate for identical masses of the $\mathrm{H}_{2} \mathrm{Ti}_{3} \mathrm{O}_{7}$ NTA and the comparison samples. Figure 6 shows that the normalized absorption $C / C_{0}(C=$ concentration at time $t, C_{0}=$ initial concentration) decays exponentially with time, which implies that the photodecomposition is a first order reaction. The calculated rate constant was the highest $\left(0.002 \mathrm{~min}^{-1}\right)$ for the supported $\mathrm{H}_{2} \mathrm{Ti}_{3} \mathrm{O}_{7}$ NTA sample. In comparison, the corresponding rate constant is $0.001 \mathrm{~min}^{-1}$ for $\mathrm{P}-25$ and $0.0003 \mathrm{~min}^{-1}$ for the $\mathrm{H}_{2} \mathrm{Ti}_{3} \mathrm{O}_{7}$ nanotube mesh. We point out that the specific surface area of the P-25 Degussa powder (as per the specification sheet) is 50 $\pm 15 \mathrm{~m}^{2} / \mathrm{g}$, which is in between the values for the substrateattached HTO sample and for free $\mathrm{H}_{2} \mathrm{Ti}_{3} \mathrm{O}_{7}$ nanotubes (see Section 3.1). However, it is well-known that the high photocatalytic activity of P-25 arises mainly from reasons other than high surface area. P-25 is a mixed phase system containing atypically small rutile crystallites intermeshed with anatase crystallites. Charges produced on rutile by visible light are stabilized through rapid electron transfer to lower energy anatase lattice trapping sites. Thus, rutile acts as antenna that extends the photoactivity into visible wavelengths and the structural arrangement of biphasic $\mathrm{TiO}_{2}$ crystallites creates catalytic "hot spots" at the rutile-anatase interface [21]. Thus, a direct quantitative comparison of P25 and HTO nanotubes based on surface area alone would be inaccurate. However, our rate constant data (Figure 6) indicates at least that the dye degradation rate of the $\mathrm{H}_{2} \mathrm{Ti}_{3} \mathrm{O}_{7}$ NTA photocatalyst compares very favorably with standard commercial photocatalysts.

Additional control experiments were performed to confirm the photocatalytic efficiency off the $\mathrm{H}_{2} \mathrm{Ti}_{3} \mathrm{O}_{7}$ NTA. The time dependence of the $\mathrm{RhB}$ solution concentration was monitored (i) on being exposed to UV irradiation without the $\mathrm{H}_{2} \mathrm{Ti}_{3} \mathrm{O}_{7}$ NTA and (ii) in presence of the $\mathrm{H}_{2} \mathrm{Ti}_{3} \mathrm{O}_{7}$ NTA but without UV irradiation. Almost no change in the $\mathrm{RhB}$ concentration was observed during either case, thereby confirming that both the $\mathrm{H}_{2} \mathrm{Ti}_{3} \mathrm{O}_{7}$ photocatalyst and the UV light are necessary for the efficient degradation of RhB. Earlier studies $[14,15]$ had reported that the rate of surface adsorption of dye (in the dark) on $\mathrm{H}_{2} \mathrm{Ti}_{3} \mathrm{O}_{7}$ nanotubes is faster than the rate of dye degradation under UV light. But our study clearly shows that the rate of dye degradation under UV is much faster than the surface adsorption in the dark. This may be ascribed to the much larger effective surface area of the nanotube array catalyst and possibly also to the greater crystallinity of the sample.

Our study has also established the following important issues. While several earlier authors-such as Hodos et al. [12] - had suggested that "undoped tri-titanate nanotubes failed to act as photocatalysts" and need to be sensitized with CdS, we have shown clear evidence of strong photocatalytic activity in this material when in the form of an array. One of the few papers that reported significant photocatalytic activity in $\mathrm{H}_{2} \mathrm{Ti}_{3} \mathrm{O}_{7}$ nanofibers [9] also found the activity to be measurably enhanced when the nanofibers were decorated with anatase nanoparticles. Zheng et al. have recently reported the synthesis of hydrogenated anatase nanotube powder by heating pristine $\mathrm{H}_{2} \mathrm{Ti}_{3} \mathrm{O}_{7}$ nanotubes in $\mathrm{H}_{2}$ atmosphere [22]. They suggest that the hydrogenated $\mathrm{TiO}_{2}$ nanotube surface shows better photocatalytic activity than bare $\mathrm{TiO}_{2}$ nanorods and $\mathrm{P}-25$. The rate constant of this sample for $\mathrm{H}_{2}$-production is 3.8 times higher than that of $\mathrm{P}-25$ and 3.2 times that of bare $\mathrm{TiO}_{2}$ nanorods. The enhanced photocatalytic activity is ascribed to improved visible light absorption and efficient separation of photogenerated excitons by the surface $\mathrm{Ti}-\mathrm{H}$ bonds.

It is instructive to compare the degradation rate for the sulforhodamine dye by the $\mathrm{H}_{2} \mathrm{Ti}_{3} \mathrm{O}_{7}$ nanofiber mesh [9] with that for the rhodamine $\mathrm{B}$ by $\mathrm{H}_{2} \mathrm{Ti}_{3} \mathrm{O}_{7}$ nanotube arrays, reported here. An extrapolation of the data in [9] indicates that the dye concentration falls to $50 \%$ in $\approx 200 \mathrm{~min}$, while in the present study it is found to fall to $50 \%$ in $\approx 300 \mathrm{~min}$. Hence, an (roughly) aligned nanotube array, anchored to a substrate, is an extremely effective structure from the point of view of catalysis. Significantly, the surface of the HTO nanotube array has a strong affinity for aqueous media. In fact, the surface exhibits superhydrophilic behavior to an approaching water drop, which is sucked up rapidly on contact (see video clip: Supplementary Material 1 available online at http://dx.doi.org/10.1155/2014/328618). Further, on recycling the $\mathrm{H}_{2} \mathrm{Ti}_{3} \mathrm{O}_{7}$ NTA catalyst, we found almost no change in the crystal structure and overall morphology-as confirmed by XRD and TEM observations (not shown here). The catalytic activity decays slightly on recycling (with 0.001 $<k<0.002$ ) but does not fall below the performance of P-25.

\section{Conclusions}

We have demonstrated that an aligned array of crystalline hydrogen titanate nanotubes acts as an effective and recyclable photocatalyst for the degradation of organic dye molecules such as rhodamine B. This is in contrast to previous 


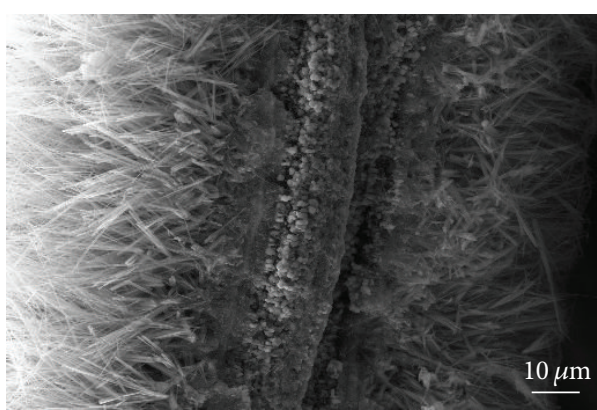

(a)

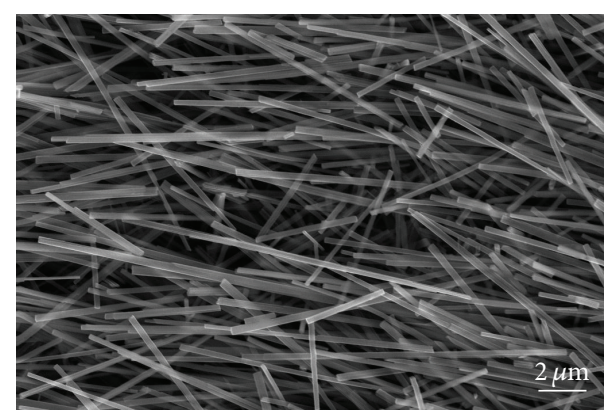

(b)

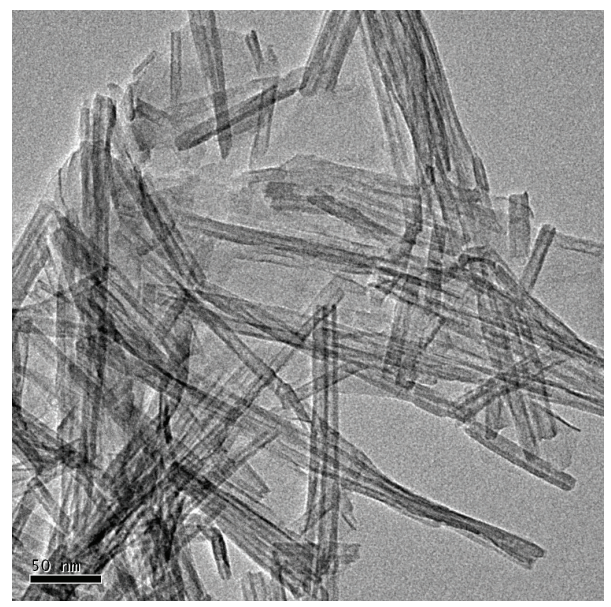

(c)

Figure 2: (a) Cross-sectional FE-SEM image of the oxidized Ti foil after hydrothermal treatment of $6 \mathrm{~h}$, showing formation of $1 \mathrm{D}$ nanostructure array on both sides of the central metal rich oxide layer. (b) Plan view of the same sample from one side. (c) HR-TEM image of a few nanotubes broken off from the same sample.

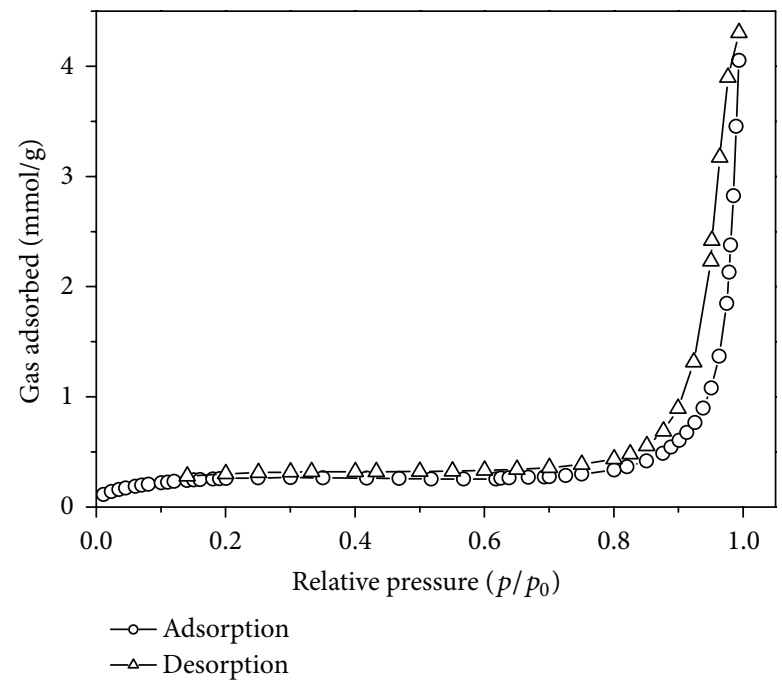

FIGURE 3: Nitrogen adsorption-desorption isotherm of an aligned array of $\mathrm{H}_{2} \mathrm{Ti}_{3} \mathrm{O}_{7}$ nanotubes.

studies on the same nanotubes in the form of a powder or mesh, which generally exhibited poor photocatalytic activity, if at all. In the present case, the fact that the self-supported

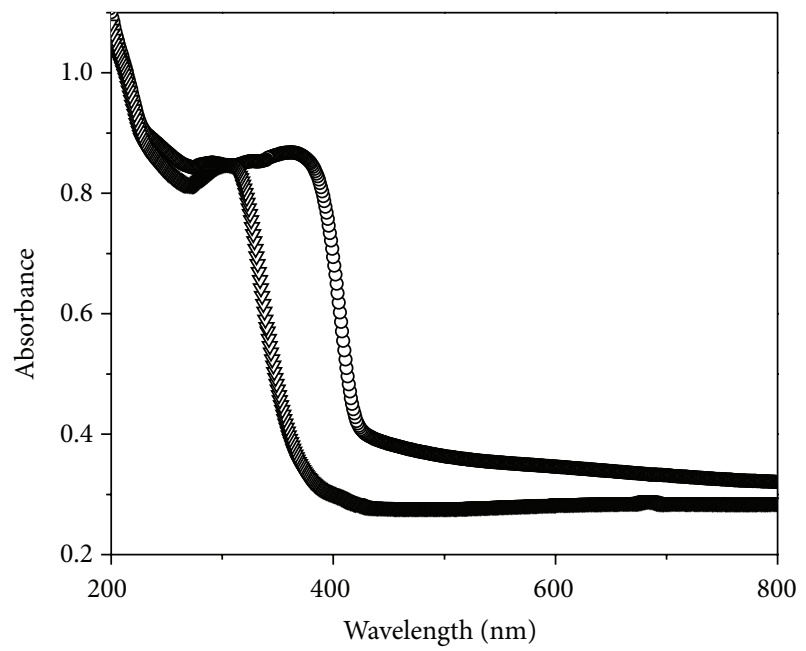

O Oxidized Ti foil

$\nabla \mathrm{H}_{2} \mathrm{Ti}_{3} \mathrm{O}_{7}$ nanotube array

FIGURE 4: Diffuse reflectance optical spectra of oxidized Ti (rutile) foil and $\mathrm{H}_{2} \mathrm{Ti}_{3} \mathrm{O}_{7}$ nanotube array.

nanotubes are permanently attached to both faces of a large area Ti foil (which can easily be as large as $10 \mathrm{~cm} \times 10 \mathrm{~cm}$ ) leads to the following obvious advantages when used as a 


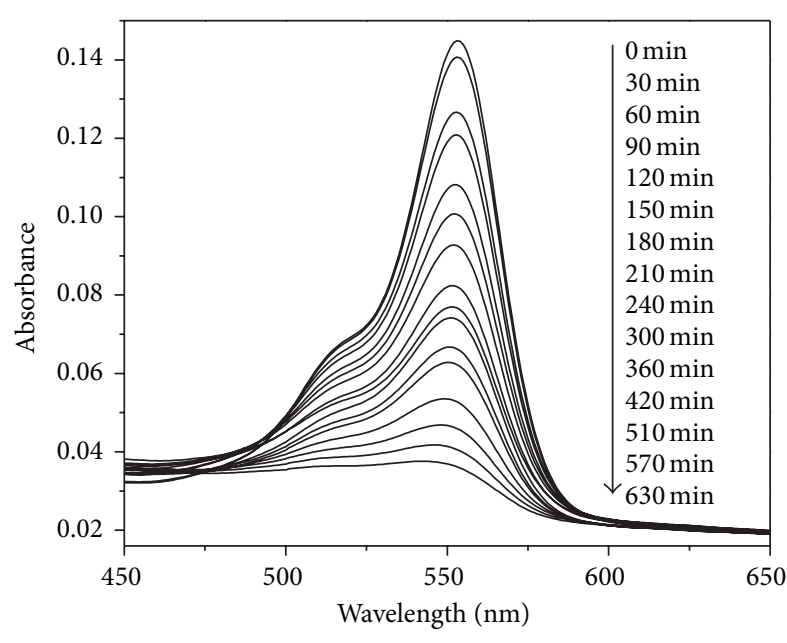

(a)

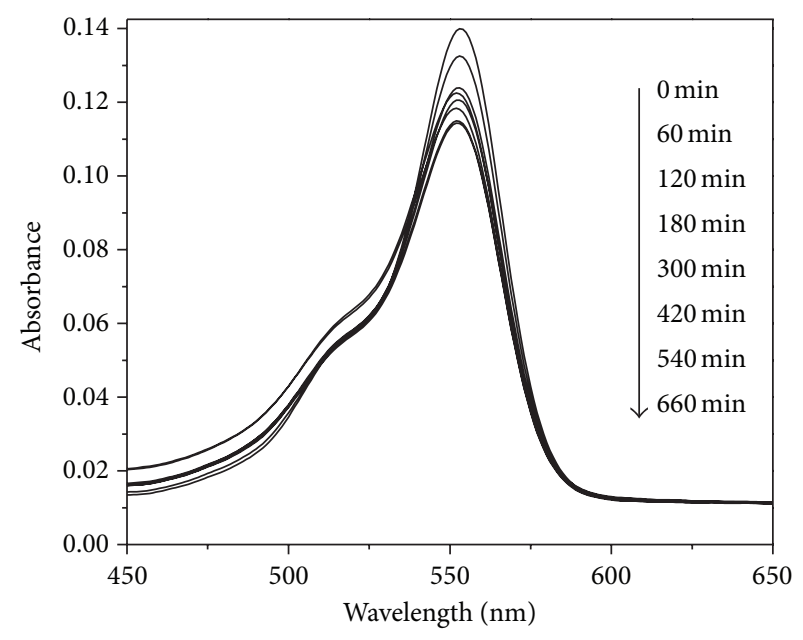

(b)

FIgURE 5: Time dependence of the optical absorption spectrum of rhodamine $\mathrm{B}$ dye solution in presence of the $\mathrm{H}_{2} \mathrm{Ti}_{3} \mathrm{O}_{7}$ nanotube array (a) and the (precursor) oxidized Ti foil (b).

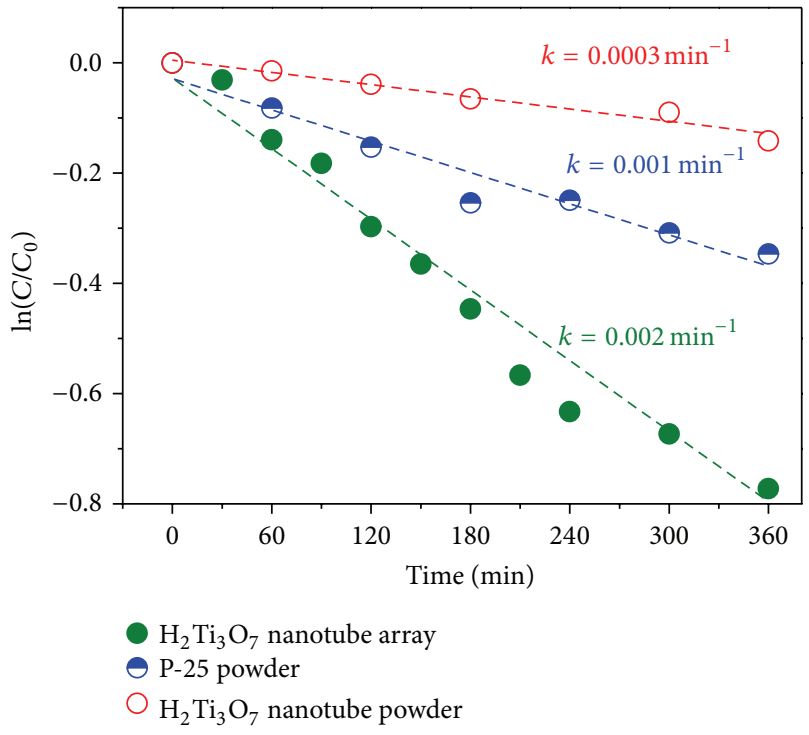

Figure 6: Photocatalytic degradation curves for $\mathrm{H}_{2} \mathrm{Ti}_{3} \mathrm{O}_{7}$ nanorod array (filled circles), P-25 Degussa (half-filled circles), and $\mathrm{H}_{2} \mathrm{Ti}_{3} \mathrm{O}_{7}$ nanoparticles (open circles), where $C / C_{0}=$ normalized absorption. The corresponding rate constants are also shown.

nanocatalyst. (1) High surface area: in the roughly aligned array, the nanotubes maintain a minimum separation, thus ensuring a higher surface area (and better catalytic activity) than in a nanotube mesh. The unique structure-with the nanotubes growing out of both faces of the central Ti foilleads to a further doubling of the exposed surface of the catalyst. (2) Superhydrophilic surface: the superhydrophilic nature of the nanotube array surface promotes rapid contact with water-soluble reactants. (3) Minimal agglomeration: being anchored at one end, the chances of nanotubes agglomerating over prolonged use are minimal. A regeneration of the catalyst by heating is a practical possibility in this case, while spherical nanoparticles would be prone to thermal aggregation. (4) High efficiency: the photodecomposition rate constant for the supported $\mathrm{H}_{2} \mathrm{Ti}_{3} \mathrm{O}_{7}$ nanotube array catalyst is comparable to or higher than those of both $\mathrm{H}_{2} \mathrm{Ti}_{3} \mathrm{O}_{7}$ nanotube powder and P-25 (Degussa). (5) Ease of handling: the central Ti foil acts as a holder for the nanotubes, making it simple to introduce and remove them from a liquid reaction bath for subsequent regeneration and reuse.

In summary, we have presented substantial data to suggest that well-crystallized, substrate-anchored $\mathrm{H}_{2} \mathrm{Ti}_{3} \mathrm{O}_{7}$ nanotube arrays-synthesized by a simple hydrothermal method-should prove to be extremely useful in important, large scale, environmental applications such as the removal of organic contaminants from wastewater with high efficiency and stability and recyclability.

\section{Conflict of Interests}

The authors declare that there is no conflict of interests regarding the publication of this paper.

\section{Acknowledgments}

The authors would like to thank Ms. B. A. Chalke for TEM studies, Dr. D. Khushalani for valuable suggestions, and Dr. M. Deshmukh and Dr. S. Bhattacharyya for their assistance during FE-SEM experiments.

\section{References}

[1] E. J. Weber, "Chemical- And sediment-mediated reduction of the azo dye disperse blue 79," Environmental Science and Technology, vol. 29, no. 5, pp. 1163-1170, 1995.

[2] V. Kulkarni, C. D. A. L. Blackard, C. W. Stackhouse, and M. W. U. S. Alexander, "Textile dyes and dyeing equipment: classification, properties and environmental aspects," US Environmental Protection Agency Report EPA-600/2-85/010, 1985. 
[3] K. Vinodgopal, D. E. Wynkoop, and P. V. Kamat, "Environmental photochemistry on semiconductor surfaces: photosensitized degradation of a textile azo dye, Acid Orange 7, on $\mathrm{TiO}_{2}$ particles using visible light," Environmental Science and Technology, vol. 30, no. 5, pp. 1660-1666, 1996.

[4] M. R. Hoffmann, S. T. Martin, W. Choi, and D. W. Bahnemann, "Environmental applications of semiconductor photocatalysis," Chemical Reviews, vol. 95, no. 1, pp. 69-96, 1995.

[5] J.-M. Herrmann, "Heterogeneous photocatalysis: fundamentals and applications to the removal of various types of aqueous pollutants," Catalysis Today, vol. 53, no. 1, pp. 115-129, 1999.

[6] R. Asahi, T. Morikawa, T. Ohwaki, K. Aoki, and Y. Taga, "Visible-light photocatalysis in nitrogen-doped titanium oxides," Science, vol. 293, no. 5528, pp. 269-271, 2001.

[7] T. Y. Peng, D. Zhao, K. Dai, W. Shi, and K. Hirao, "Synthesis of titanium dioxide nanoparticles with mesoporous anatase wall and high photocatalytic activity," Journal of Physical Chemistry B, vol. 109, no. 11, pp. 4947-4952, 2005.

[8] S. Parra, S. E. Stanca, I. Guasaquillo, and K. Ravindranathan Thampi, "Photocatalytic degradation of atrazine using suspended and supported $\mathrm{TiO}_{2}$," Applied Catalysis B, vol. 51, no. 2, pp. 107-116, 2004.

[9] H. Zhu, X. Gao, Y. Lan, D. Song, Y. Xi, and J. Zhao, "Hydrogen titanate nanofibers covered with anatase nanocrystals: a delicate structure achieved by the wet chemistry reaction of the titanate nanofibers," Journal of the American Chemical Society, vol. 126, no. 27, pp. 8380-8381, 2004.

[10] A. Fujishima, X. Zhang, and D. A. Tryk, " $\mathrm{TiO}_{2}$ photocatalysis and related surface phenomena," Surface Science Reports, vol. 63, no. 12, pp. 515-582, 2008.

[11] J. Li, Z. Tang, and Z. Zhang, "Layered hydrogen titanate nanowires with novel lithium intercalation properties," Chemistry of Materials, vol. 17, no. 23, pp. 5848-5855, 2005.

[12] M. Hodos, E. Horváth, H. Haspel, Á. Kukovecz, Z. Kónya, and I. Kiricsi, "Photosensitization of ion-exchangeable titanate nanotubes by CdS nanoparticles," Chemical Physics Letters, vol. 399, no. 4-6, pp. 512-515, 2004.

[13] F. Amano, T. Yasumoto, T. Shibayama, S. Uchida, and B. Ohtani, "Nanowire-structured titanate with anatase titania: characterization and photocatalytic activity," Applied Catalysis $B$, vol. 89, no. 3-4, pp. 583-589, 2009.

[14] K. V. Baiju, S. Shukla, S. Biju, M. L. P. Reddy, and K. G. K. Warrier, "Hydrothermal processing of dye-adsorbing onedimensional hydrogen titanate," Materials Letters, vol. 63, no. 11, pp. 923-926, 2009.

[15] C. K. Lee, K. S. Lin, C. F. Wu, M. D. Lyu, and C. C. Lo, "Effects of synthesis temperature on the microstructures and basic dyes adsorption of titanate nanotubes," Journal of Hazardous Materials, vol. 150, no. 3, pp. 494-503, 2008.

[16] S. Chatterjee, K. Bhattacharyya, P. Ayyub, and A. K. Tyagi, "Photocatalytic properties of one-dimensional nanostructured titanates," Journal of Physical Chemistry C, vol. 114, no. 20, pp. 9424-9430, 2010.

[17] H. Fu, C. Pan, W. Yao, and Y. Zhu, "Visible-light-induced degradation of rhodamine $\mathrm{B}$ by nanosized $\mathrm{Bi}_{2} \mathrm{WO}_{6}$," Journal of Physical Chemistry B, vol. 109, no. 47, pp. 22432-22439, 2005.

[18] S. Chatterjee, S. Bhattacharyya, D. Khushalani, and P. Ayyub, "Hydrothermally synthesized aligned arrays of self-assembled multiwalled hydrogen titanate nanotubes," Crystal Growth and Design, vol. 10, no. 3, pp. 1215-1220, 2010.
[19] M. Ray, S. Chatterjee, T. Das, S. Bhattacharyya, P. Ayyub, and S. Mazumdar, "Conjugation of cytochrome $c$ with hydrogen titanate nanotubes: novel conformational state with implications for apoptosis," Nanotechnology, vol. 22, no. 41, Article ID 415705, 2011.

[20] D. V. Bavykin, J. M. Friedrich, and F. C. Walsh, "Protonated titanates and $\mathrm{TiO}_{2}$ nanostructured materials: synthesis, properties, and applications," Advanced Materials, vol. 18, no. 21, pp. 2807-2824, 2006.

[21] D. C. Hurum, A. G. Agrios, K. A. Gray, T. Rajh, and M. C. Thurnauer, "Explaining the enhanced photocatalytic activity of Degussa P25 mixed-phase $\mathrm{TiO}_{2}$ using EPR," Journal of Physical Chemistry B, vol. 107, no. 19, pp. 4545-4549, 2003.

[22] Z. Zheng, B. Huang, J. Lu et al., "Hydrogenated titania: synergy of surface modification and morphology improvement for enhanced photocatalytic activity," Chemical Communications, vol. 48, pp. 5733-5735, 2012. 

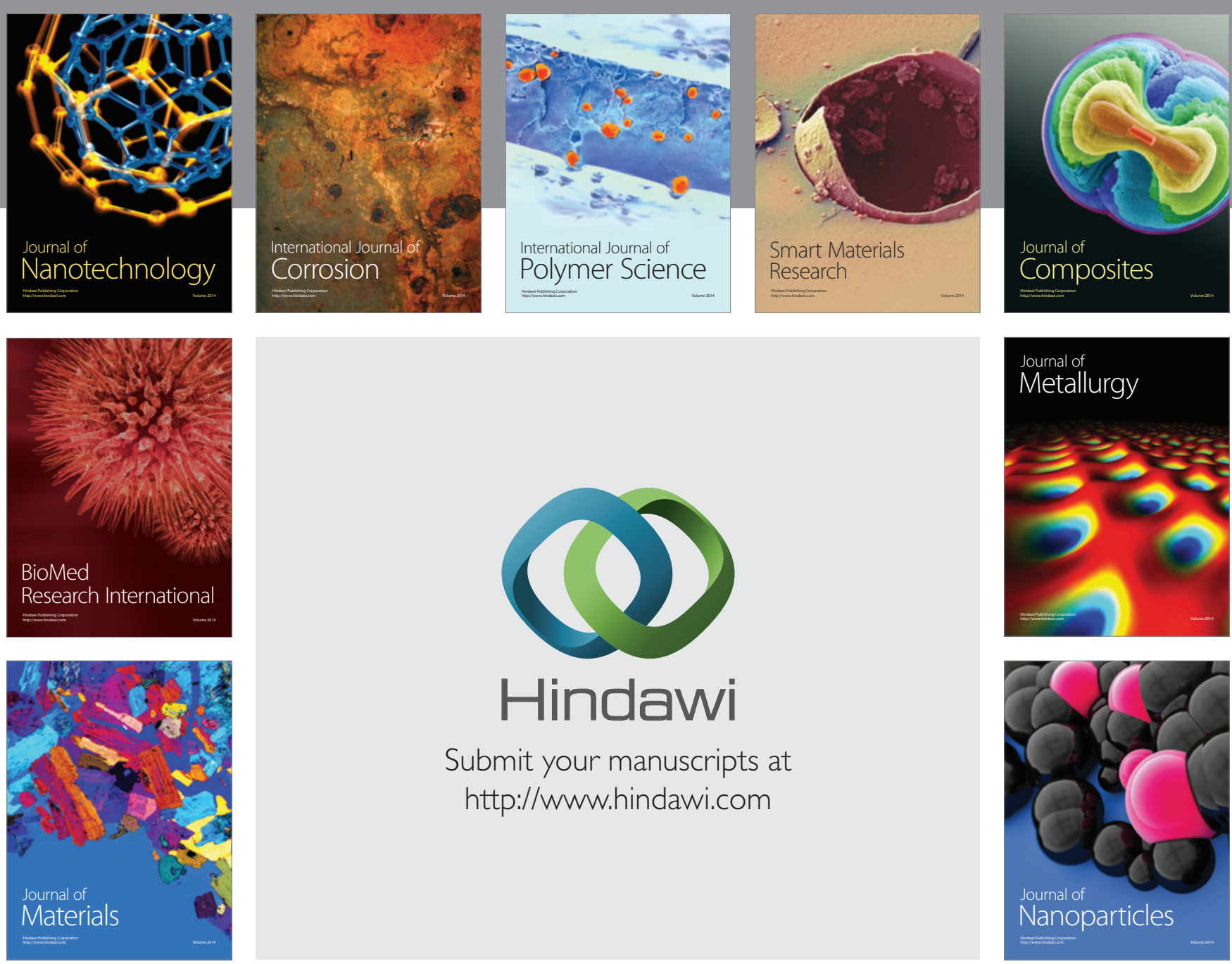

Submit your manuscripts at http://www.hindawi.com
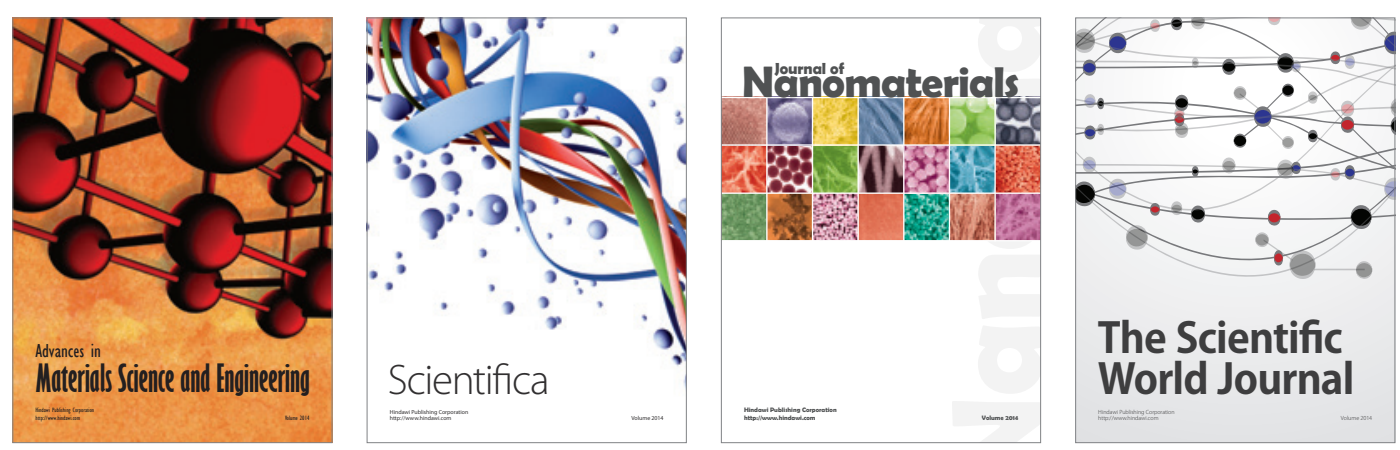

\section{The Scientific World Journal}
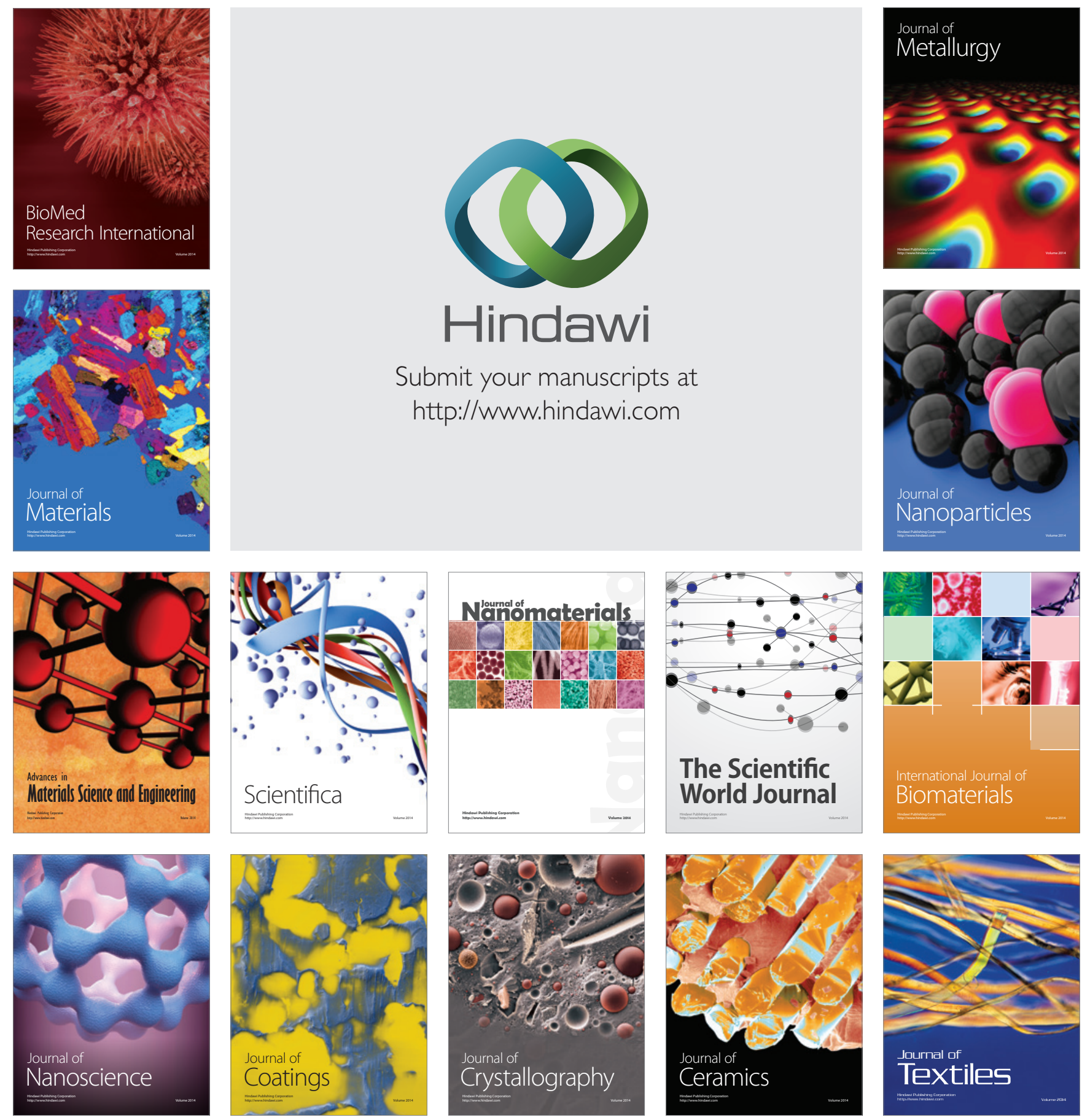